\title{
Value of prehospital assessment of spine fracture by paramedics
}

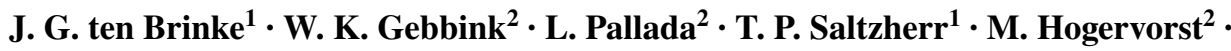 \\ J. C. Goslings ${ }^{1}$
}

Received: 23 March 2017 / Accepted: 1 August 2017 / Published online: 5 August 2017

(c) The Author(s) 2017. This article is an open access publication

\begin{abstract}
Background Current guidelines state that trauma patients at risk of spine injury should undergo prehospital spine immobilization to reduce the risk of neurological deterioration. Although this approach has been accepted and implemented as a standard for decades, there is little scientific evidence to support it. Furthermore, the potential dangers and sequelae of spine immobilization have been extensively reported. The role of the paramedic in this process has not yet been examined. The aim of this study was to evaluate the accuracy of prehospital evaluations for the presence of spine fractures made by paramedics.

Methods All patients who presented with prehospital spine immobilization at our level II trauma center between January 2013 and January 2014 were prospectively included in a database. Prior to the diagnosis, paramedics recorded the probability of a spine fracture after a prehospital examination. These predictions were compared with patient outcomes. The sensitivity, specificity, positive predictive value, and negative predictive value were calculated.

Results One hundred and thirty-nine patients were included that positive predictive value was $22 \%$, negative predictive value was $95 \%$, sensitivity was $92 \%$, specificity was $30 \%$, and accuracy was $41 \%$.

Conclusions The results of this study suggest that paramedics cannot accurately predict spinal fractures.
\end{abstract}

\section{J. G. ten Brinke}

j.g.tenbrinke@amc.nl

1 Trauma Unit, Department of Surgery, Academic Medical Center, Meibergdreef 9, 1105 AZ Amsterdam, The Netherlands

2 Department of Surgery, Gelre Hospital Apeldoorn, Apeldoorn, The Netherlands
Keywords Spinal fracture - Emergency medical services · Prehospital $\cdot$ Predictive value

\section{Introduction}

Current worldwide guidelines state that trauma patients at risk of spine injury should be immobilized by emergency medical services to reduce the risk of neurological deterioration [1-5]. The reason for these immobilization precautions is the assumption that unstable spinal injuries can deteriorate due to manipulation or movement, thereby causing secondary injury to the spinal cord $[2,6,7]$. This treatment algorithm has been accepted and implemented as the standard of care for decades, despite their being little scientific evidence to support this practice [8-12]. More than five million patients in the United States receive spinal immobilization each year [13]. The majority of blunt trauma patients do not have a spine fracture, meaning that many patients are immobilized unnecessarily. Spine immobilization can be problematic for both the patient and the paramedic: it can cause pressures sores, compromise respiration, necessitate aspiration after vomiting, raise intracranial pressure, and hamper airway management [14-17]. It is also a time-consuming intervention [17]. Development of a more selective immobilization protocol could reduce the number of immobilized patients, thereby decreasing the potential dangers and sequelae associated with unnecessary spine immobilization. Many of these selective immobilization protocols were initially designed for indications requiring radiological imaging in emergency rooms and later validated as a prehospital immobilization protocol [18-21].

Although previous research has shown that paramedics can accurately predict injury severity [22-24], the accuracy of spine fracture prediction has not yet been investigated. 
The purpose of this study was to answer the following question: how accurate can paramedics predict the presence of a spinal fracture?

\section{Methods}

\section{Study design}

This was a single institution prospective cohort study. Approval for this study was obtained from the medical ethical committee of Gelre Hospital.

\section{Patients and setting}

All patients that presented at the emergency department of our level II trauma center with prehospital immobilization between January 2013 and January 2014 were included in a database.

\section{Data collection}

Before radiologic imaging, paramedics recorded the probability of a spine fracture based on their own evaluation on a data collection form. They were asked to predict any spine fractures by answering 'Yes' or 'No'. The paramedics also recorded the mechanism of injury (MOI). Patients were assessed according to the Advanced Trauma Life Support (ATLS) guidelines [25] and spinal imaging was performed according to Dutch guidelines [26]. The presence of a spine fracture was ruled out if computed tomography scanning was negative or if no clinical symptoms suggesting a spinal injury were detected during the 3-month follow-up period. Exclusion criteria were the absence of paramedic's prediction and/or lack of appropriate imaging. Patients were stratified based on the prehospital prediction of a spine fracture.

\section{Primary outcome}

Sensitivity, specificity, positive predictive value (PPV), negative predictive value (NPV), and accuracy were calculated.

\section{Data analysis}

Data were analyzed using SPSS for Windows, Version 21.0 (SPSS Inc., Chicago, IL, USA).

\section{Results}

A total of 190 patients presented with prehospital spine immobilization and 139 of these patients were included in this study (Fig. 1). The baseline characteristics and MOI

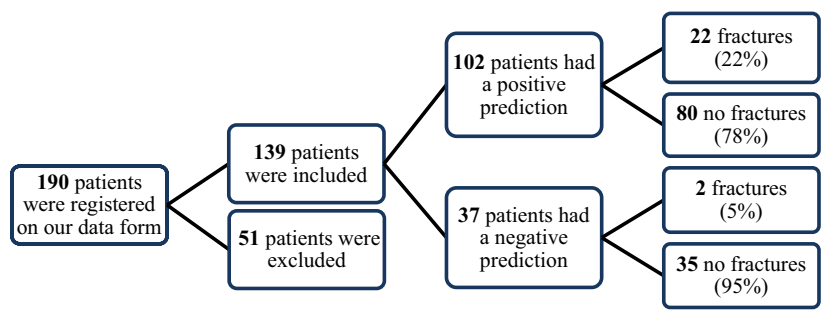

Fig. 1 Flow diagram of patient inclusion and fracture prediction

are presented in Table 1. The prevalence of spine fractures was $17 \%$. Paramedics failed to predict spine fractures in two patients (92\% sensitivity and 30\% specificity). The PPV was $22 \%$ and the NPV was $95 \%$. The accuracy was $41 \%$. The primary outcome and accuracy of this study are presented in Table 2. Twenty-two patients of the 102 were correctly predicted as having spinal fractures by the paramedics. The paramedics failed to identify spine fractures in two patients. The mechanism of injury in these patients with missed fractures was a fall from height. One of these patients had multiple spinal fractures and was given an orthesis (Table 3 ). Thirty-five patients were immobilized by the paramedics according to protocol, despite there being no suspicion of a spinal fracture by the paramedics (Table 2).

\section{Discussion}

This study demonstrates that paramedics can predict the presence of a spinal fracture with a low degree of accuracy, as demonstrated by the accuracy of $41 \%$ found here. The decision to perform prehospital spine immobilization is currently based on PreHospital Trauma Life Support (PHTLS) criteria, which were previously reported to have an accuracy of $66 \%$ [19]. This figure was supported by a systematic review conducted in 2012 [22]. The low accuracy of the prehospital evaluations conducted by the paramedics in the current study suggests that implementation of a protocol based on paramedics predictions will not reduce the overuse of spine immobilization. The sensitivity in this study (92\%) is similar to earlier findings from [19], which demonstrates that a paramedic-prediction approach will not limit sensitivity compared with current protocols. Michaleff et al. also reported a similar sensitivity to our study, although their investigation was limited to cervical spine fractures [22].

In the present study, we found that spine fractures are falsely predicted in $70 \%$ of trauma patients. Although this specificity of $30 \%$ seems low, it is in line with the results of previous studies that have looked at current guidelines [19, $22,27]$. Furthermore, the NPV of paramedic spine fracture evaluations reported here (95\%) is the same as that reported in the current protocol for spine immobilization $[1,19]$. 
Table 1 Baseline characteristics stratified by prehospital prediction

\begin{tabular}{lll}
\hline & Positive prediction & Negative prediction \\
\hline Number & 102 & 37 \\
Median age $\left(\mathrm{IQR}^{\mathrm{a}}\right)$ & $41(22-60)$ & $23(18-46)$ \\
Male & $60 \%$ & $70 \%$ \\
MOI $^{\mathrm{b}}$ & & \\
High-energy trauma $_{\text {Isolated blunt trauma }}$ & $4(41 \%)$ & $17(46 \%)$ \\
$\begin{array}{l}\text { Lateral impact on verte- } \\
\text { bral column }\end{array}$ & $5(5 \%)$ & $11(30 \%)$ \\
Other high-energy trauma & $12(12 \%)$ & $3(8 \%)$ \\
\hline
\end{tabular}

${ }^{\text {a }}$ Inter quartile range: 25 and 75 th percentile

${ }^{\mathrm{b}}$ Mechanism of injury

Table 2 Prehospital prediction of a spine fracture by paramedics

\begin{tabular}{llcc}
\hline & \multicolumn{2}{l}{ Definitive diagnosis } & Total \\
\cline { 2 - 3 } & Fracture & No fracture & \\
\hline $\begin{array}{c}\text { Paramedic predic- } \\
\text { tion: fracture }\end{array}$ & 22 & 80 & 102 \\
$\begin{array}{c}\text { Paramedic predic- } \\
\text { tion: no fracture }\end{array}$ & 2 & 35 & 37 \\
\begin{tabular}{l} 
Total \\
\hline
\end{tabular} & 24 & 115 & 139 \\
\hline
\end{tabular}

Table 3 Details of spine fractures in patients with a negative prediction

\begin{tabular}{llll}
\hline Patient & MOI & Fracture type & Treatment \\
\hline Male, 46 years & $4 \mathrm{~m}$ fall & T7 compression & Pain relief \\
Male, 69 years & $2.5 \mathrm{~m}$ fall & T11, L2, L4 compression & Orthesis \\
\hline
\end{tabular}

MOI Mechanism of Injury

Paramedics failed to predict spine fractures in two patients (5\%), mainly because the symptoms were mild at initial presentation and a painful distracting injury was present. At the 3-month follow-up, only one of these patients still had symptoms, while the other had fully recovered. None of these patients sustained spinal cord injury.

One limitation of this study was the high number of exclusions. The main reason for exclusion was the absence of a completed data form. Another limitation of our study is that we did not consider how many years of experience the paramedics had; it is possible that more experienced paramedics can more accurately predict spinal fractures in trauma patients. In addition, patients did not receive a CT scan of the whole spine, only of the segments that had an indication for this type of imaging. Some spine fractures may, therefore, have been missed, although the clinical relevance of a missed fracture is questionable. The fact that this study was performed in a level II trauma center means that patients with extreme severe trauma and a high chance of positive prediction are excluded. The high prevalence of spine fractures in this study (17\%) relative to the prevalence found in the literature (3-5\%) [28] could be caused by how we selected the patients in our study. We selected all patients that had undergone prehospital immobilization according to our national protocol. However, recent numbers of spinal fractures are increasing due to osteoporosis and the increased use of computed tomography scans [29].

This study could be added to by further investigating how accurately paramedics can predict the location of spine fractures and whether cervical, thoracic, or lumbar fractures are easiest to predict. Future studies should analyze a larger number of paramedics and take the fracture location and years of experience into account.

\section{Conclusion}

Paramedics cannot accurately predict spinal fractures based on the trauma mechanism and clinical symptoms.

\section{Compliance with ethical standards}

Conflict of interest Joost ten Brinke, Wieke Klein Gebbink, Len Pallada, Teun Saltzherr, Mike Hogervorst, and Carel Goslings have no conflict of interest. The manuscript is not under consideration for publication elsewhere.

Human and animal rights All procedures followed were in accordance with the ethical standards of the responsible committee on human experimentation (institutional and national) and with the Helsinki Declaration of 1975, as revised in 2008.

Informed consent Informed consent was not obtained from all patients for inclusion in the study because of the design of this study, namely, an observational database study.

Open Access This article is distributed under the terms of the Creative Commons Attribution 4.0 International License (http://creativecommons.org/licenses/by/4.0/), which permits unrestricted use, distribution, and reproduction in any medium, provided you give appropriate credit to the original author(s) and the source, provide a link to the Creative Commons license, and indicate if changes were made.

\section{References}

1. Landelijk protocol ambulancezorg versie 8,1. 2016.

2. PHTLS: Prehospital Trauma Life Support. 8 ed.

3. Kreinest M, et al. Development of a new Emergency Medicine Spinal Immobilization Protocol for trauma patients and a test of applicability by German emergency care providers. Scand J Trauma Resusc Emerg Med. 2016;24:71.

4. Theodore N, et al. Prehospital cervical spinal immobilization after trauma. Neurosurgery. 2013;72(Suppl 2):22-34.

5. Unfallchirurgie DGf. S3-Leitlinie Polytrauma/SchwerverletztenBehandlung. 2011. 
6. Crosby ET. Tracheal intubation in the cervical spine-injured patient. Can J Anaesth. 1992;39(2):105-9.

7. Benger J, Blackham J. Why do we put cervical collars on conscious trauma patients? Scand J Trauma Resusc Emerg Med. 2009; $17: 44$.

8. Orledge JD, Pepe PE. Out-of-hospital spinal immobilization: is it really necessary? Acad Emerg Med. 1998;5(3):203-4.

9. Kwan I, F Bunn, I Roberts. Spinal immobilisation for trauma patients. Cochrane Database Syst Rev. 2001.

10. Hauswald M, et al. Out-of-hospital spinal immobilization: its effect on neurologic injury. Acad Emerg Med. 1998;5(3):214-9.

11. Hood N, Considine J. Spinal immobilisaton in pre-hospital and emergency care: a systematic review of the literature. Australas Emerg Nurs J. 2015;18(3):118-37.

12. Sundstrom T, Asbjørnsen H, Habiba S, Sunde GA, Wester K. Prehospital use of cervical collars in trauma patients: a critical review. J Neurotrauma. 2014;31:531-40.

13. Frohna WJ. Emergency department evaluation and treatment of the neck and cervical spine injuries. Emerg Med Clin North Am. 1999;17(4):739-91.

14. Chan D, et al. The effect of spinal immobilization on healthy volunteers. Ann Emerg Med. 1994;23(1):48-51.

15. Chendrasekhar A, Moorman DW, Timberlake GA. An evaluation of the effects of semirigid cervical collars in patients with severe closed head injury. Am Surg. 1998;64(7):604-6.

16. Cordell $\mathrm{WH}$, et al. Pain and tissue-interface pressures during spine-board immobilization. Ann Emerg Med. 1995;26(1):31-6.

17. Morrissey J. Spinal immobilization. Time for a change. JEMS. 2013;38(3):28-30 (32-36, 38-39).

18. Domeier RM, et al. Multicenter prospective validation of prehospital clinical spinal clearance criteria. J Trauma. 2002;53(4):744-50.
19. Domeier RM, Frederiksen SM, Welch K. Prospective performance assessment of an out-of-hospital protocol for selective spine immobilization using clinical spine clearance criteria. Ann Emerg Med. 2005;46(2):123-31.

20. Burton JH, et al. A statewide, prehospital emergency medical service selective patient spine immobilization protocol. J Trauma. 2006;61(1):161-7.

21. Vaillancourt $\mathrm{C}$, et al. The out-of-hospital validation of the Canadian C-Spine Rule by paramedics. Ann Emerg Med. 2009;54(5):663-71.

22. Michaleff ZA, et al. Accuracy of the Canadian C-spine rule and NEXUS to screen for clinically important cervical spine injury in patients following blunt trauma: a systematic review. CMAJ. 2012;184(16):E867-76.

23. Mulholland SA, et al. Is paramedic judgement useful in prehospital trauma triage? Injury. 2005;36(11):1298-305.

24. Lavoie A, et al. Evaluation of the Prehospital Index, presence of high-velocity impact and judgment of emergency medical technicians as criteria for trauma triage. CJEM. 2010;12(2):111-8.

25. Advanced trauma life support, 10th edn.

26. NO V. Richtlijn acute traumatische wervelletsels. 2009.

27. Baez AA, Schiebel N. Evidence-based emergency medicine/ systematic review abstract. Is routine spinal immobilization an effective intervention for trauma patients? Ann Emerg Med. 2006;47(1):110-2.

28. Burney RE, et al. Incidence, characteristics, and outcome of spinal cord injury at trauma centers in North America. Arch Surg. 1993;128(5):596-9.

29. ten Brinke JG, et al. Incidence of spinal fractures in the Netherlands 1997-2012. J Clin Orthop Trauma (In press). 